\title{
APPLICATION OF ANALYTIC HIERARCHY PROCESS TO DETERMINING A PRIORI DISTRIBUTION OF ERROR-FREE RUNNING TIME FOR HIGH- RELIABILITY COMPONENTS
}

\author{
Nikolai N. Radaev \\ Limited Liability Company \\ Scientific-Research Institute of Natural Gases \\ and Gas Technologies - Gazprom VNIIGAZ, \\ Moscow, Russia \\ Alexander V. Bochkov* \\ Limited Liability Company \\ Scientific-Research Institute of Natural Gases \\ and Gas Technologies - Gazprom VNIIGAZ, \\ Moscow, Russia \\ E-mail: a.bochkov@gmail.com
}

\begin{abstract}
We have considered the approach to determining a priori distribution of error-free running time for highreliability components by the method of paired comparisons useful for the increase of their reliability indicators. The fuzzy variables are introduced. The degrees of membership of these fuzzy variables are interpreted as subjective probability of finding the error-free running time in different time intervals. The method of recording the expert evaluation accuracy has been suggested.
\end{abstract}

Keywords: AHP, priori distribution, error-free running time, high-reliability components, expert evaluation accuracy

\section{Introduction}

To control the meeting of the requirements by such high-reliability components as components of nuclear reactors (Ostreikovsky, Salnikov, 1990), aircraft and space-and-missile engineering (Radaev, 1997), gas equipment (Mitrofanov, 2007), etc., it is necessary to evaluate small (below 0.01) failure probabilities for the preset error-free running time. When there is practically no statistics on the failures of these components during their operation, the error-free running time distribution law is required for evaluating the reliability indicators with acceptable accuracy. This permits, in particular, the subsequent use of information pooling techniques (Melnikov, Severtsev, 1987), for instance, Bayesian methods of pooling the a priori information and observational data (Savchuk, 1989).

Determination of error-free running time distribution belongs to intricately formalized problems (there are no sufficiently accurate mathematical models for its solution in most cases (Ostreikovsky, Salnikov, 1990), (Bolotin, 1984)). There is also no sufficiently representative statistics on the failures of highreliability components (Radaev, 1997). Therefore, to obtain the a priori distribution of error-free running time for a component, it is expedient to employ expert evaluation (Vishnyakov, Radayev, 2007). The necessity of using non-formal experience and appreciating the physical nature of failures is also caused by

* Corresponding author 
the fact that, as is shown by simulation modeling of various distribution laws, small samples with the same mean values may result in considerable differences in description of distribution tail areas, which substantially influences on the accuracy of determining the reliability indicators of high-reliability components.

The aim of the article consists in employing expert evaluation for finding out the type and parameters of distribution of error-free running time for high-reliability components.

\section{Problem formulation}

Let $F(t)=P(T<t)$ be the law of distribution of error-free running time, $T$ be the random value of error-free running time for a component. Select on the time axis $\mathrm{n}$ periods, within which the failures of the component under consideration are expected. During the operation a random component from a certain main entity may fail in the $z$-th $(z=1,2, \ldots, n)$ period, namely, its possible state. The error-free running time is associated with the discrete state of the component by relationship

$$
t=t_{0}+\Delta t \cdot z
$$

where $t_{0}$ is the maximum error-free running time, until which the component failures have not been observed yet; $\Delta t$ is the duration of the time period corresponding to the discrete state of the component.

Then, discrete random value $Z$ described by means of bar chart $\tilde{f}_{Z}(z)$ corresponds to continuous variate $T$ of the component with probability density function $f_{T}(t)$. In its turn, continuous variate $T$ can be made to correspond to discrete random value $Z$ set by any method.

It is necessary to obtain the expected distribution of the component's error-free running time.

\section{Solution method}

The problem of obtaining bar chart $\tilde{f}_{Z}(z)$ can be solved by the method of paired comparisons ( a na lys is of h i e ra r chi es ) ( Radaev, 2007)) developed by T. Saaty (Saaty, 2008). All pairs of the component's states are presented to the expert and the latter each time determines which of them is preferable with respect to a possibility of finding the component's error-free running time. In the course of assessment the experts take into account the following: available data on all kinds of reliability tests of the component and its failures during the operation; own experience in evaluating the reliability indicators of similar components by various methods and other factors. The evaluation process results in paired comparisons matrix $B=\left(b_{i j}\right)$ where

$$
b_{i j}=\left\{\begin{array}{l}
2 \text { if } i \succ j ; \\
1 \text { if } i \approx j ; \quad(i, j=1, \ldots, n), \\
0 \text { if } i \prec j ;
\end{array}\right.
$$

where $i, j$ are the component's compared states of $n$ possible ones. 


\section{Radaev N., Bochkov A. / Application of AHP to Determining a Priori Distribution of Error-Free Running Time for High-Reliability Components}

The constituents of normalized maximum characteristic vector $q$ of paired comparisons matrix $B$ are taken to be relative weights characterizing a possibility of the component's staying in each state.

The method modification, namely, the method of paired comparisons based on a qualitative attribute with quantitative preference judgment. In the course of paired comparisons and fillingin of matrix $B$ the expert not only selects the preferable state in each pair, but also indicates how many times this state is preferable with respect to a possibility of finding the error-free running time than in another state of the pair. The method does not require compulsory transitivity of the expert's preferences, while the processing of the paired comparisons matrices is easily realizable on computers. However, the method has no clear physical interpretation and is unable to treat obtained evaluations $q$ as subjective probabilities (Mitropolsky, 1971). This hampers a possibility of employing the conceptual and mathematical apparatuses well developed in the theory of probability and mathematical statistics for further operations with the obtained results. Therefore, let us complement the method with a fuzzy model (Cox, Hinkly, 1978).

Let us introduce the following indistinct variables:

1) "Possible error-free running time" (basic) - for evaluating the possibility of finding the error-free running time, i.e., probability density function $f_{T}(t)$.

Auxiliary indistinct variables can be also introduced for solving such subproblems as calibration clarification of the parameters obtained with the aid of the first indistinct distribution variable, evaluation of the expert's assessment accuracy, etc.;

2) “Expected error-free running time” - for evaluating average error-free running time $t_{c p}$;

3) “Most probable error-free running time" - for evaluating the distribution mode.

Let the considered indistinct variable be determined on discrete multitude $Z=\{z\}$ from $n$ possible component states. Indistinct multitude $\tilde{Z}$ on multitude $Z$ appears to be an aggregate of pairs $\tilde{Z}=\left\{\left\langle\mu_{\mathrm{Z}}(z) / z\right\rangle\right\}$ where $\mu_{\mathrm{z}}(z)$ is the function of the error-free running time's membership in the fuzzy set, whose sense is formalized by the chosen indistinct variable. The function of membership is made up of degrees of membership (relative weights $q_{z}$ ) of states $z \in Z$ in multitude $\tilde{Z}$. We shall treat them as subjective probabilities of finding error-free running time $z$. Meant by the subjective probability is the estimate of probability (relative weight) of finding the error-free running time within a certain time period obtained as a result of processing the experts' opinions rather than mathematically on the basis of the statistic data on frequency of failures getting into this time period as it happens in case of objective probability.

Greater values of $\mu_{\mathrm{z}}(\mathrm{z})$ correspond to the states conforming, to greater extent, to the meaning of the chosen indistinct variable (i.e., with a greater possibility of finding the component's error-free running time in these state and time period).

As usually $n \geq 4$, the approximated method (Melikhov, Bershtein, Korovin, 1990) is recommended for finding the normalized maximum characteristic vector. To do this, introduced is normalized characteristic vector $q^{(r)}=B q^{(r-1)}$ of the paired comparisons matrix where $r$ is the No. of the approximate computation algorithm's step.

Then, let us assume that the relative weights are represented by the constituents of the normalized vector at the $r$-th iteration step determined from the formula 


$$
\hat{q}^{(r)}=B q q_{(r 1)}^{(r 1)} / \lambda^{(r)},
$$

where $\lambda^{(r)}$ is the sum of the constituents of vector $B q$ or

$$
\hat{q}_{i}^{(r)}=\frac{1}{\lambda^{(r)}} \sum_{j=1}^{m} b_{i j} \hat{q}_{i}^{(r-1)} ; \quad \lambda^{(r)}=\sum_{i=1}^{m} \sum_{j=1}^{m} b_{i j} \hat{q}_{j}^{(r-1)}
$$

till reaching preset accuracy $\varepsilon$. The required accuracy of calculation of the characteristic vector constituents is preset beforehand (henceforth we accept $\varepsilon=0.0001$ for further computations) and the calculation is stopped at step $r$ if $n$ conditions $\left|\hat{q}_{i}^{r-1}-\hat{q}_{i}^{r}\right| \leq \varepsilon \quad \forall i$ are satisfied.

\section{Procedure of expert evaluation and interpretation of its results}

It is expedient to begin expert evaluation by the selected method from plotting the function of the possible error-free running time's membership in the fuzzy set, whose meaning is formalized by the first indistinct variable. To do this, first one should indicate the range of the component's possible error-free running time: monad $\left\langle t_{0}, \Delta t, n\right\rangle$ expected in the initial approximation. Paired comparisons matrix $B$ is obtained by way of interrogating the experts on the extent, to which, in their opinion, state $i$ corresponds to the meaning of the "Possible error-free running time" indistinct variable more than state $j$. When matrix $B$ is filled in, the expert compares, with the aid of the scale developed by T. Saaty (Saaty, 2008) (Table 1), all pairs of discrete states with respect to a possibility of the component failure in them. So, to allot marks $b_{i j}$, the expert actually compares the expected densities of probability of finding the error-free running time (frequency of failures) during various time periods.

Table 1. Modified paired comparisons scale developed by T. Saaty

\begin{tabular}{|c|l|l|}
\hline $\begin{array}{c}\text { Preference } \\
\text { degree } b_{i j}\end{array}$ & \multicolumn{1}{|c|}{ Definition } & \multicolumn{1}{c|}{ Explanation } \\
\hline 1 & $\begin{array}{l}\text { States are } \\
\text { equally likely }\end{array}$ & $\begin{array}{l}\text { Possibility of finding the (average or most probable) }{ }^{*} \text { component's error-free running time } \\
\text { in both time periods of the compared pair is similar }\end{array}$ \\
\hline 2 & \multicolumn{2}{|c|}{ Intermediate meaning } \\
\hline 3 & $\begin{array}{l}\text { Poor } \\
\text { superiority }\end{array}$ & $\begin{array}{l}\text { The expert's experience makes it possible to consider the possibility of finding the } \\
\text { (average or most probable) }{ }^{*} \text { component's error-free running time in the first time period of } \\
\text { the pair somewhat higher than in the second one }\end{array}$ \\
\hline 4 & \multicolumn{2}{|c|}{ Intermediate meaning $^{\mid}$} \\
\hline 5 & $\begin{array}{l}\text { Strong } \\
\text { superiority }\end{array}$ & $\begin{array}{l}\text { The expert considers that the possibility of finding the (average or most probable) } \\
\text { component's error-free running time in the first time period of the pair is definitely higher } \\
\text { than in the second one }\end{array}$ \\
\hline
\end{tabular}

\footnotetext{
${ }^{*}$ Here and hereinafter in Table 1 the text in brackets pertains to either second or third indistinct variable.
} 
Table 1 (continued)

\begin{tabular}{|c|c|c|}
\hline $\begin{array}{l}\text { Preference } \\
\text { degree } b_{i j}\end{array}$ & Definition & Explanation \\
\hline 6 & & Intermediate meaning \\
\hline 7 & $\begin{array}{l}\text { Apparent } \\
\text { superiority }\end{array}$ & $\begin{array}{l}\text { The expert considers that the possibility of finding the (average or most probable) } \\
\text { component's error-free running time in the first time period of the pair is apparently higher } \\
\text { than in the second one, while the available statistics of failures of the analyzed } \\
\text { components under the similar conditions, as well as the model calculations conform this } \\
\text { fact }\end{array}$ \\
\hline 8 & & Intermediate meaning \\
\hline 9 & $\begin{array}{l}\text { Absolute } \\
\text { superiority }\end{array}$ & $\begin{array}{l}\text { The expert has no doubts with respect to the fact that the possibility of finding the } \\
\text { (average or most probable) }{ }^{*} \text { component's error-free running time in the first time period of } \\
\text { the pair is absolutely higher than in the second one }\end{array}$ \\
\hline
\end{tabular}

As a result of processing matrix $B$ we shall obtain function $\mu_{\mathrm{z}}(\mathrm{z})$ of the error-free running time's membership in fuzzy set $\widetilde{Z}$, the meaning of which is formalized by indistinct variable "Possible error-free running time". The membership function is formed by the membership degrees, which can be represented by the components of the normalized maximum characteristic vector of matrix $B$. Let us interpret this function of this function as the bar chart of the observed random value of the component's error-free running time, including the error of its expert evaluation. This bar chart can help determining the kind and parameters of the observed distribution of error-free running time and, in particular, giving an approximated estimate of observed average error-free running time $\tilde{m}_{c p}^{\prime}=\sum_{z=1}^{n} z \cdot \tilde{f}(z)$ and observed errorfree running time dispersion $\left(s^{\prime}\right)^{2}=\sum_{z=1}^{n}\left(z-\tilde{m}_{c p}^{\prime}\right)^{2} \cdot \tilde{f}(z)$

By changing over to $t$ according to (1), we shall respectively obtain

$$
\tilde{t}_{c p}^{\prime}=\sum_{z=1}^{n} t_{z} \cdot \tilde{f}(z)
$$

and

$$
\left(s_{t}^{\prime}\right)^{2}=\sum_{z=1}^{n_{1}}\left(t-\tilde{t}_{c p}^{\prime}\right)^{2} \cdot \tilde{f}(z)
$$

where $t_{z}$ is the error-free running time meaning corresponding to the middle of the $z$-th time period.

\section{Example 1.}

The expert is asked to evaluate the component's error-free running time in seven time periods, each 2 years long, beginning from the $6^{\text {th }}$ year of its operation. Paired comparisons matrix $B$ from the expert's judgments pertinent to distribution of the possible component's error-free running time is shown in Fig. 1(a).

Determine the type and parameters of the form of the expected error-free running time. 


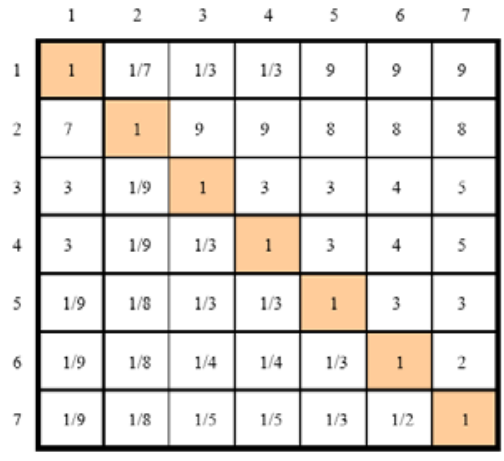

(a)

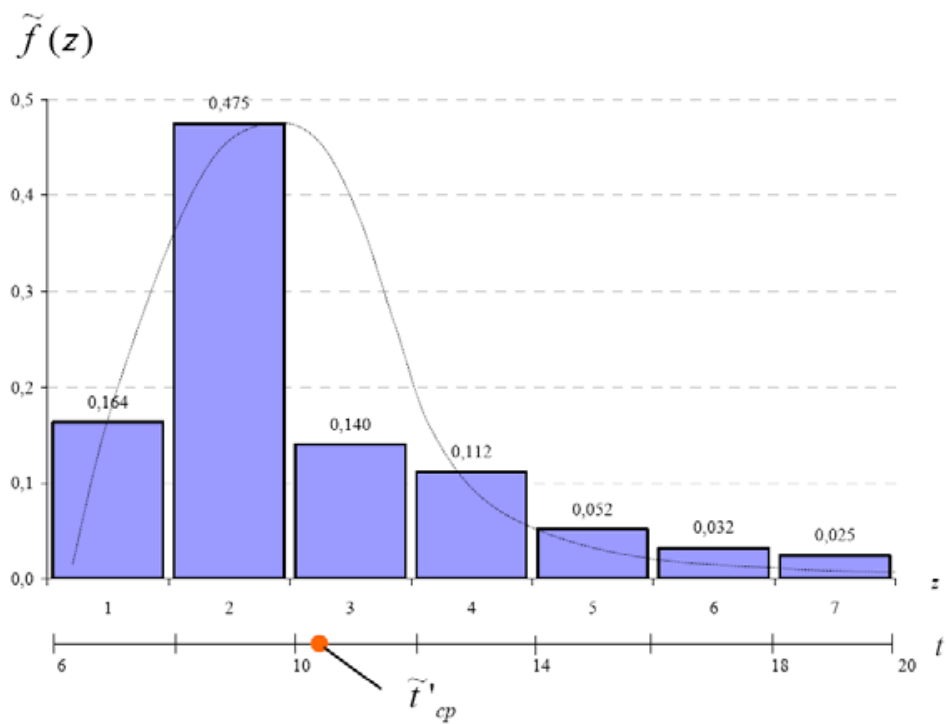

(b)

Figure 1. Paired comparisons matrix of possible component's error-free running time (a) and respective bar chart of observed error-free running time (b).

\section{Solution:}

As a result of processing matrix $B_{1}$ we use the approximative method to obtain the components of normalized characteristic vector $q_{z}$, having the meaning of relative weights (probabilities) of finding the component within certain time periods $z$ on time axis $t$, i.e. bar chart $\tilde{f}(z)$ of the component's error-free running time in Fig. 1(b).

The average error-free running time is found from formula (2) and amounts to $\tilde{t}_{c p}^{\prime}=7 \cdot 0.164+9 \cdot 0.475+$ $11 \cdot 0.140+13 \cdot 0.112+15 \cdot 0.052+17 \cdot 0.032+19 \cdot 0.025=10.22$ years, while the root-mean-square deviation with allowance for (3) amounts to $s_{t}{ }^{\prime}=3.99$ years.

The type of a priori distribution of error-free running time corresponding to the bar chart obtained by the expert method can be determined by various methods: method of moments, with the aid of statistical criteria, etc. However, the use of known goodness-of-fit tests (Pearson's, Kolmogorov's, Shapiro's, Wilk's, Bartlett's test, Mann's, etc.) requires getting the answer to the question: to which number of statistic observations do the results of expert evaluation of the error-free running time by the group of experts correspond? Therefore, let us use the method of moments.

When balancing the "statistical" rows by this method, use is often made of the system of Pearson curves (Mitropolsky, 1971). The values of the coordinates obtained in the form of distribution bar chart $f_{T}(t)$ in the diagram (Fig. 2) make up $\beta_{1}=1.641$ and $\beta_{2}=4.250$. So, the distribution of error-free running time by the expert method obtained can be adjusted by distribution from the family of $J$-shaped beta distributions (Fig. 2). 


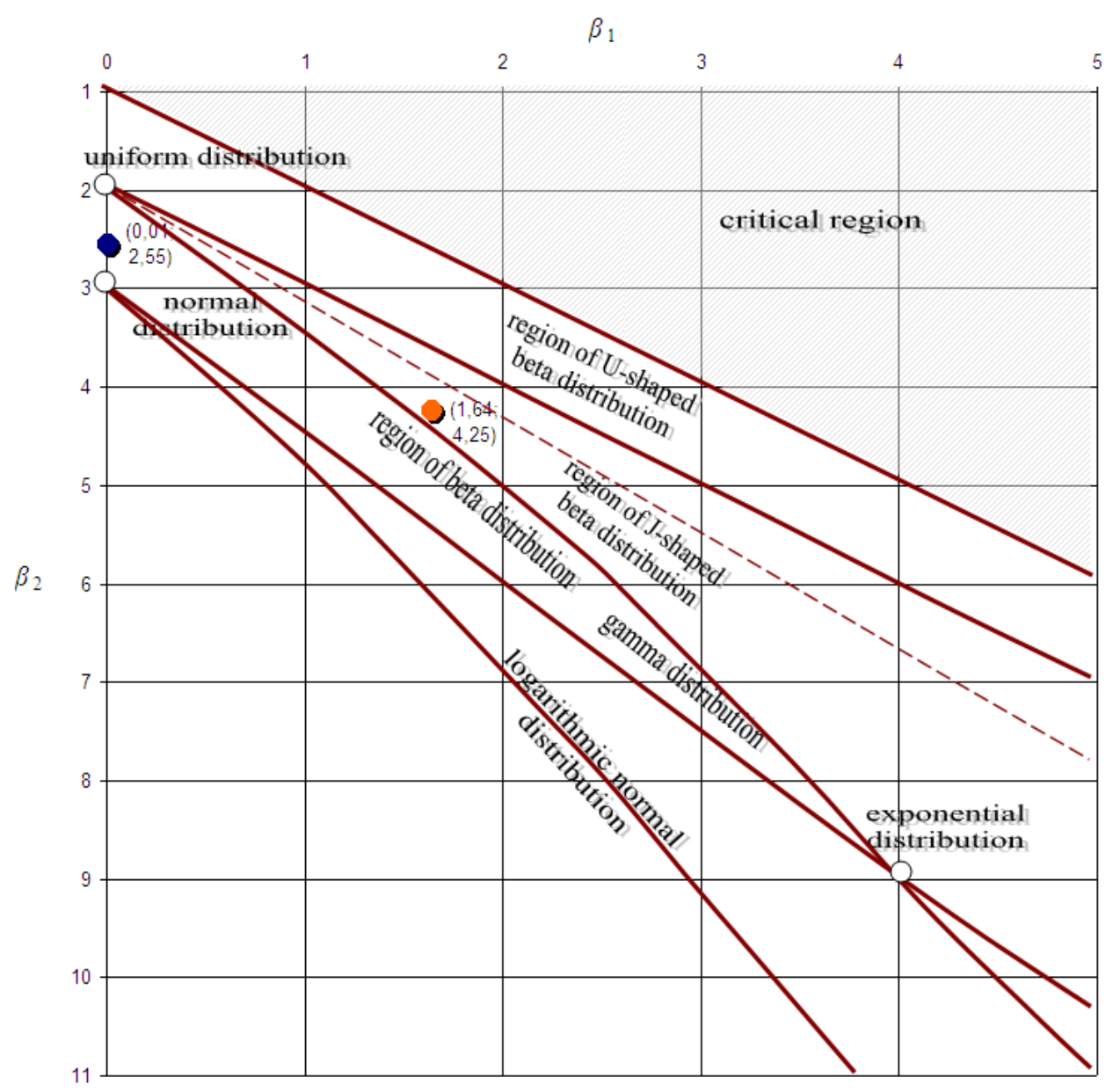

Figure 2. Location of obtained distribution on diagram of distributions of family of Pearson curves.

Now let us consider the "Expected error-free running time" indistinct variable and indicate to the experts the range of its possible values $\left\langle t_{2}, \Delta t_{2}, n_{2}\right\rangle$ with the aid of information on $\tilde{t}_{c p}^{\prime}$ and bar chart $\tilde{f}_{T}(t)$. During the repeated evaluation the experts compare the pairs of possible time periods with respect to the possibility of finding the average error-free running time within them. As a result of processing paired comparisons matrix $B_{2}$ formed in such a manner, we shall obtain bar chart $\tilde{f}_{2}(z)$ with discrete random value $Z_{2}$ of the average error-free running time (Fig. $3 \mathrm{~b}$ ). This bar chart is helpful in specifying the estimate of the average error-free running time:

$$
\tilde{t}_{c p}=\sum_{z=1}^{n_{2}} t_{z} \cdot \tilde{f}_{2}(z)
$$

and obtain the dispersion of its evaluation

$$
s_{c p}^{2}=\sum_{z=1}^{n_{2}}\left(t_{z}-\tilde{t}_{c p}\right)^{2} \cdot \tilde{f}_{2}(z)
$$




\section{Example 2.}

Basing on the data of Example 1 for assessing the average error-free running time, let us choose $n_{2}=5$ time periods, beginning from the $9^{\text {th }}$ year of the component's operation, half a year long each. Obtained as a result of expert evaluation is paired comparisons matrix $B_{2}$ for a possibility of finding the average errorfree running time within these time periods (Fig. $3 \mathrm{~b}$ ).

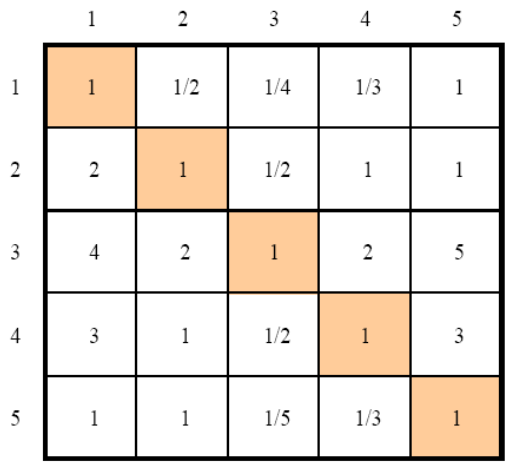

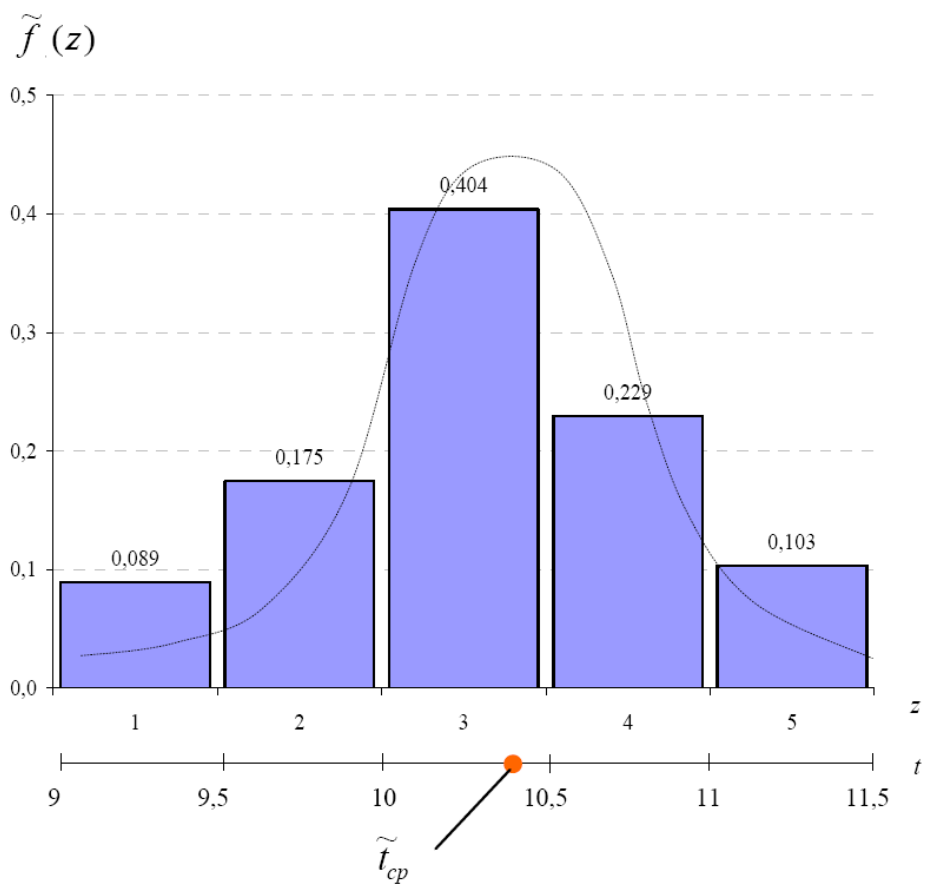

(a)

(b)

Figure 3. Paired comparisons matrix (a) and bar chart of average error-free running time (b) obtained as a result of expert evaluation.

It is necessary to determine the component's average error-free running time and accuracy of its evaluation by the expert.

Solution:

Let us obtain the bar chart of the average error-free running time (Fig. 3 b) by the approximative method. Take estimation of expectation $m_{c p}=M\left[Z_{2}\right]$ of discrete random variable $Z_{2}$ as the average error-free running time. While changing over to variable $t$, we obtain the specified estimate of the average errorfree running time

$$
\tilde{t}_{c p}=\sum_{z=1}^{n_{2}} t_{z} \cdot \tilde{f}_{2}(z)=9.25 \cdot 0.089+9.75 \cdot 0.175+10.25 \cdot 0.404+10.75 \cdot 0.229+11.25 \cdot 0.103=10.29 \text { years. }
$$

The root-mean-square deviation of this estimate with allowance for (5) will amount to 0.15 year. 


\section{Radaev N., Bochkov A. / Application of AHP to Determining a Priori Distribution of Error-Free Running Time for High-Reliability Components}

The values of the coordinates for the system of Pearson curves determined from bar chart $f_{2}(t)$ make up $\beta_{1}=0.010$ and $\beta_{2}=2.553$ (see Fig. 2). Their position in the diagram shows that the distribution of the average error-free running time estimate obtained by the expert method is predictably close to the normal one.

So, as a result of expert evaluation with the aid of the first indistinct variable, when the experts compare the selected time periods with respect to the possibility of the component's failure during it, bar chart $\tilde{f}_{T}(t)$ of the component's error-free running time was obtained and, with its aid of the information on dispersion of the component's error-free running time. However, the measurement errors are imposed on the results of expert evaluation of the error-free running time.

The expert (by convention, a highly knowledgeable specialist) acts as a "measuring instrument” for expert evaluation. Therefore, bar chart $\tilde{f}_{T}(t)$ describes observed component's error-free running time $T^{\prime}$ obtained with the aid of expert evaluation. Let us assume that the dispersion of the error-free running time with respect to the average value determined by the expert method includes two additive constituents:

$$
\Delta^{\prime}=\Delta+\Delta^{e}
$$

where $\Delta$ is the actual dispersion of the error-free running time, which should be taken into account in determining the component's reliability indicators; $\Delta^{\mathrm{e}}$ is the expert evaluation error.

The problems of accuracy of the expert methods are discussed in (Litvak, 2004). However, to assess accuracy, use is made, as a rule, of indirect indicators not relying on the characteristics employed in the theory of accuracy. It is obvious that accuracy of expert evaluation of the component's error-free running time depends on the quality of experts, namely, their competence, objectivity, and information awareness. The a priori and a posteriori assessment of the expert (Litvak, 2004) can be done with the aid of usually interrelated indicators:

- "weight" of the expert normalized with respect to other experts under a certain rule (the expert's "weight” depends on his education, academic degree, knowledge of physics of the component's failures, practical experience in determination of the reliability indicators) and set by the decision of the "absolutely competent" person - a priori estimate;

- accuracy of the estimates made by the experts - a posteriori estimate.

If we consider the expert to be the measuring instrument, to analyze accuracy of expert evaluation, generally accepted metrological performances serve turn. Of them the most universal one is root-meansquare deviation $\sigma^{e}$ of the "measurement" result relative to the true (or average - in the absence of systematic errors) meaning. Accuracy of the estimates expressed in terms of value $\sigma^{e}$ can be determined by the following methods:

- by deviations of the expert estimates from the true meaning. This method is implemented by testing the experts on the problems with the a priori known result or with the result instrumentally (statistically) determined after expert evaluation. The method advantage consists in exclusion of systematic errors, while the disadvantage, in considerable expenses;

- by means of the dispersion characteristics ("concentration") of the obtained expert estimates relative to the true (average) meaning. This method is also applicable in the situations, when the true state of the object being evaluated is unknown, but it does not take into account possible systematic errors. 
The disadvantage of both methods consists in the necessity of a certain sample for finding the dispersion.

Let us assume that the characteristic of accuracy of expert evaluation of the error-free running time under the second method is represented by the root-mean-square deviation in determining a certain fixed state [14]. We shall consider the average error-free running time as such a state in the problem being solved. The method advantage consists in the fact that it is obtained in case of determining a single considered component. The more contradictory and inconsistent the expert's judgment on the possible component's state, the higher the value of $\sigma^{e}$. As the dispersion meaning depends on the expert's quality, therefore it can serve as the measure of this quality.

Under the conditions of the considered example $\sigma^{e}=s_{a v}=0.15$ year.

If the expert evaluation error is known, in some cases it can be taken into account. On the condition of satisfying supposition (6)

$$
\Delta=\Delta^{\prime}-\Delta^{e}
$$

Allowance for the errors introduced by the expert is possible by way of correcting the parameters of the shape of distribution often functionally bound with the value of $\sigma$. Table 2 contains certain distributions of the error-free running time with indication of correlations required to eliminate the measurement errors.

Table 2. Correlations for elimination of expert evaluation error for certain distribution laws of error-free running time.

\begin{tabular}{|c|c|c|}
\hline Distribution law & Kind of $F(t)$ & $\begin{array}{c}\text { Correlation for correcting the form } \\
\text { parameter }\end{array}$ \\
\hline Uniform & $\frac{t-t_{a}}{t_{b}-t_{a}}$ & $\begin{array}{l}t_{a}=t_{c p}-\sqrt{3\left[\left(\sigma^{\prime}\right)^{2}-\left(\sigma^{e}\right)^{2}\right]} \\
t_{b}=t_{c p}+\sqrt{3\left[\left(\sigma^{\prime}\right)^{2}-\left(\sigma^{e}\right)^{2}\right]}\end{array}$ \\
\hline \multicolumn{3}{|c|}{ Unimodal } \\
\hline Normal & $\Phi\left\{\frac{t-m}{\sigma}\right\}$ & $\sigma=\sqrt{\left(\sigma^{\prime}\right)^{2}-\left(\sigma^{e}\right)^{2}}$ \\
\hline \multicolumn{3}{|c|}{ Unimodal with positive skew } \\
\hline$\alpha$ - distribution & $\Phi\left\{\alpha_{0}\left(1-\frac{1}{a t}\right)\right\}$ & $\alpha_{0}=m / \sqrt{\left(\sigma^{\prime}\right)^{2}-\left(\sigma^{e}\right)^{2}}$ \\
\hline Weibullized $(b \geq 1)$ & $1-\exp \left\{-\left(\frac{t-c}{a}\right)^{b}\right.$ & $\left.b=\left[\frac{1}{m} \sqrt{\left[\left(\sigma^{\prime}\right)^{2}-\left(\sigma^{e}\right)^{2}\right.}\right]\right]$ \\
\hline Logarithmic-normal & $\Phi\left\{\frac{y-m_{y}}{\sigma_{y}}\right\}$ & $\sigma_{y}=\sqrt{\left[\left(\sigma_{y}{ }^{\prime}\right)^{2}-\left(\sigma_{y}{ }^{e}\right)^{2}\right]}$ \\
\hline
\end{tabular}

* The following designations are taken in Table 2: $\Phi(\cdot)$ is the Laplace's function; $m=M[T]$ is the expectation; $a, b$, and $c$ are the scale, form, and shift of the Weibull distribution, respectively; $v$ is the coefficient of variation, $\alpha_{0}=1 / v$; $t_{a}, t_{b}$ are the uniform distribution parameters; $y=\lg t ; m_{y}=M[\lg T] ; \sigma_{y}=D[\lg T]^{1 / 2} ; \sigma_{y}^{\prime}=D\left[\lg T^{\prime}\right]^{1 / 2} ; \sigma_{y}^{\jmath}=D\left[\lg \Delta^{s}\right]^{1 / 2}$. 


\section{Radaev N., Bochkov A. / Application of AHP to Determining a Priori Distribution of Error-Free Running Time for High-Reliability Components}

\section{Conclusion}

The suggested approach makes it possible to improve the extent of justification of setting the a priori distribution of the components' error-free running time for provision of acceptable accuracy of determination of reliability indicators for high-reliability components.

\section{REFERENCES}

Ostreikovsky V.A., Salnikov N.L. (1990) Probabilistic Forecasting of Operational Capability of Power Reactor Facility Components - Moscow: ENERGOATOMIZDAT Publishers, 415 pp.

Radaev N.N. (1997) Methods of Assessing Compliance of Technical Systems with Raised Requirements at Low Scope of Testing. - Moscow: RF MoD, 390 pp.

Mitrofanov A.V. (2007) Methods of Controlling Production Machinery State by Failure Probability and Risk Criteria. - Moscow: NEDRA-BIZNESTSENTR Publishers, 380 pp.

Reliability and Efficiency in Technology. Reference guide in 10 volumes / Vol. 4: Similarity Methods in Reliability / Under general editorship of Melnikov V.A., Severtsev N.A. (1987) - Moscow: MASHINOSTROYENIYE Publishers, 280 pp.

Savchuk V.P. (1989) Bayesian Methods of Statistical Estimation. Reliability of Technical Objects. Moscow: NAUKA Publishers, 328 pp.

Bolotin V.V. (1984) Forecasting of Service Life of Machines and Structures. - Moscow: MASHINOSTROYENIYE Publishers, 312 pp.

Vishnyakov Ya.D., Radaev N.N. (2007) General Theory of Risks. - Moscow: AKADEMIA Publishers, $368 \mathrm{pp}$.

Radaev N.N. (2007) Accuracy of expert evaluation of object state by paired comparisons method with quantitative preference judgment // Izmeritelnaya Tekhnika (Instrumentation), No. 9, pp. 6- 11.

Saaty T.L. (2008) Decision-Making at Dependent Feedbacks. Analytical Networks. - Translated from English / Scientific editor A.V. Andreichikov, O. N. Andreichikova. - Moscow: LKI Publishers, 360 pp.

Cox D., Hinkly D. (1978) Theoretical Statistics / Translated from English - Moscow: MIR Publishers.

Melikhov A.N., Bershtein L.S., Korovin S.Ya. (1990) Situational Advising Systems with Fuzzy Logic. Moscow: NAUKA Publishers, 272 pp.

Mitropolsky A.K. (1971) Method of Statistical Distributions. - Moscow: NAUKA Publishers, 576 pp.

Litvak B.G. (2004) Expert Technologies in Management. - Moscow: DELO Publishers.

Radayev N.N., Zyulina A.S. (2006) Expert technologies of management: improvement of objectivity // Proceedings of VIII International Research and Practice Conference on "Quality of distance education. Concepts, problems, solutions". - Moscow, pp. 252-258. 